\title{
Gilles SIOUFFI (dir.), Une histoire de la phrase française des Serments de Strasbourg aux écritures numériques
}

\section{Sophie Jollin-Bertocchi}

\section{OpenEdition}

\section{Journals}

Édition électronique

URL : https://journals.openedition.org/scolia/1544

DOI : $10.4000 /$ scolia. 1544

ISSN : 2677-4224

\section{Éditeur}

Presses universitaires de Strasbourg

\section{Édition imprimée}

Date de publication : 9 juillet 2021

Pagination : 188-192

ISBN : 979-10-344-0091-1

ISSN : $1253-9708$

Référence électronique

Sophie Jollin-Bertocchi, « Gilles Sıouffi (dir.), Une histoire de la phrase française des Serments de Strasbourg aux écritures numériques », Scolia [En ligne], 35 | 2021, mis en ligne le 09 juillet 2021, consulté le 13 juillet 2021. URL : http://journals.openedition.org/scolia/1544 ; DOI : https://doi.org/ 10.4000/scolia.1544

\section{(C) $(0 \otimes)$}

Les contenus de la revue Scolia sont mis à disposition selon les termes de la Licence Creative Commons Attribution - Pas d'Utilisation Commerciale - Partage dans les Mêmes Conditions 4.0 International. 
Gilles SIOUFFI (dir.), Une histoire de la phrase française des Serments de Strasbourg aux écritures numériques, Arles, Actes Sud, 2020, 375 pages.

Dans la lignée de l'Histoire de la langue française de Brunot (1905-1938) et de ses continuateurs, après avoir collaboré à Mille ans de langue française: histoire d'une passion (Rey éd., 2007), Siouffi propose un nouvel ouvrage de haute vulgarisation, illustré, écrit par des spécialistes reconnus, abordant la langue sous l'angle de la phrase, et dont la parution est quasi simultanée avec celle de la Grande Grammaire Historique du Français, avec trois des mêmes auteurs. Parmi d'autres possibles, comme le suggère l'actualisation indéfinie du titre, l'approche, largement contextualisée, envisage la dimension socio-culturelle de la langue, embrassant pour ce faire non seulement la littérature, où se forgent des pratiques à la fois historicisées et singulières, mais l'ensemble des genres d'écrits. Le volume est divisé en six chapitres suivant l'ordre chronologique.

Dans le premier chapitre, "Du latin tardif au Moyen Âge: les débuts de la phrase française» ( $\mathrm{IX}^{\mathrm{e}}{ }^{-\mathrm{XIII}^{\mathrm{e}}}$ siècles), Marchello-Nizia met d'abord en évidence l'influence du cadre sonore de la littérature et du cadre versifié sur la phrase médiévale, au moment où le français se dégage progressivement de la syntaxe latine. Le français écrit va s'écarter de l'oral en opérant la segmentation des mots et groupes de mots (au moyen des blancs), et la délimitation des unités supérieures (proposition et phrase). La prose médiévale se caractérise par une "grande souplesse de la phrase [...] organisée essentiellement pour mettre en valeur l'information» (p.25), permettant ainsi l'invention des dislocations et des clivages syntaxiques. Deux facteurs conditionnent la structure phrastique: la forme (vers ou prose) et le style narratif ou discursif. L'émergence du style d'auteur dès le Moyen Âge inaugure la tradition d'une phrase littéraire, au sens de singulière et subversive, tandis que par ailleurs les "phrases popularisées » que sont les proverbes vont servir «de matrices à une forme de stylistique laconique» (p. 65) qui parcourt la littérature française postérieure.

Combettes se penche sur les siècles suivants $\left(\mathrm{XIV}^{\mathrm{e}}-\mathrm{XVI} \mathrm{I}^{\mathrm{e}}\right)$ : «Du moyen français à la Renaissance: phrase et développement de la prose». À la faveur du développement de la lecture silencieuse, le «texte n'est plus rédigé comme une suite d'énoncés destinés à être 
oralisés mais comme un tout cohérent $[\ldots]$ »(p. 74). De nouveaux outils syntaxiques (participe présent détaché et relatif de liaison) participent de ce souci de la dimension textuelle. Du latin subsiste le modèle périodique des articulations logiques et de la subordination plutôt que l'équilibre général de l'énoncé. Dans la phrase narrative en plein essor, la valorisation de l'arrière-plan du récit s'opère au moyen de la subordination et des participes. En poésie, la syntaxe s'adapte à la métrique ou l'inverse. Ainsi la virtuosité technique prime chez les "grands rhétoriqueurs", qui créent de nouvelles structures (enjambement). L'émergence d'une réflexion grammaticale sur les unités composant la phrase mène à une uniformisation progressive des pratiques de ponctuation et à l'introduction du point-virgule, tandis que les questions de syntaxe restent marginales, entre la grammaire soucieuse surtout de morphologie et de catégories de mots, et la rhétorique.

Dans le troisième chapitre, "Entre phrase et période», Siouffi retrace comment le XviI ${ }^{\mathrm{e}}$ siècle a été le cadre d'une "révolution de la phrase» (p. 136) et des débuts de la littérarisation du style, autorisant une phrase écrite qui ne soit plus nécessairement oratoire. La prose narrative se caractérise désormais par la coexistence de deux modèles (phrase longue et phrase courte) issus de la dichotomie oratoire (asianiste $v s$ atticiste), au moment où le vers classique se rigidifie en un système métrique très codifié, « essayant de faire coïncider les frontières du vers et les frontières de la syntaxe» (p. 138). Le mot phrase signifie alors "expression", "manière de parler» et désigne les expressions figées d'une langue. La segmentation de la période rhétorique et l'autonomisation possible de ses unités constituent «l'étape décisive vers la phrase au sens moderne» (p. 153). Au moment où la période est envisagée sous un angle plus grammatical, la Grammaire de PortRoyal établit un parallèle entre la structure logique de la proposition et sa structure syntaxique, début d'une longue tradition d'analyse rationaliste. L'émancipation de la phrase courte est incarnée par le style coupé, la "conversationnalisation» des genres de discours favorisant l'apparition d'une phrase française simple, symbolique de la «tentation de perfection».

Le chapitre suivant, "L'invention de la phrase moderne» (XVIII ${ }^{\mathrm{e}}$ siècle), du même auteur que le précédent, aborde la période de 
théorisation de la phrase. La séquence «sujet-verbe-objet» est érigée en principe linguistique au nom d'une prétendue clarté d'expression de la pensée (Rivarol), qui permet une apologie de la langue française. Parmi les grammairiens qui s'essaient à de nouvelles définitions de la phrase pour dissiper la confusion avec la proposition, Domergue construit une typologie des propositions qui lui permet de distinguer la phrase longue de la période et marque le «triomphe de la grammaire face à la rhétorique» (p. 189). La phrase des écrivains se veut plus subjective et plus libreà travers l'affaiblissement de la distinction vers/prose et des constructions nouvelles (association d'un nom à un verbe support de sens large faire, donner, prendre). Face à la phrase symbolisant l'ordre et la contrainte, la Révolution construit une nouvelle culture de l'écrit dont témoigne la phrase infinie des "peu-lettrés». Par ailleurs, le deuxpoints et le point-virgule deviennent les ponctuants fondamentaux dans les textes narratifs et argumentatifs du XVIII ${ }^{\mathrm{e}}$ siècle, parmi d'autres formes nouvelles d'éloquence.

«La phrase à l'heure de l'enseignement» (XIX ${ }^{\mathrm{e}}$ siècle), écrit par Dürrenmatt, porte une grande attention à la ponctuation et accentue l'approche stylistique. Le mythe de la clarté française n'est plus une question d'esthétique mais une nécessité sociale. Dans cette optique, le point «acquiert [...] une valeur capitale de délimitation» (p. 231) et la pensée de la hiérarchisation qui sous-tend la phrase a pour conséquences deux modifications importantes de la norme grammaticale: l'«affirmation d'impératifs dans la position des propositions les unes par rapport aux autres» et la raréfaction des constructions à détachement. Le «tiraillement» entre phrase longue et phrase courte porte préjudice aux signes de ponctuation intermédiaires. La syntaxe naturelle et émotive repose sur l'inversion et la ponctuation (points de suspension et tirets). Dans les "phrases non conformes» (étrangères, régionales, orales ou intérieures), "la logique énonciative prime sur la logique grammaticale» (p. 247). La ponctuation est désormais perçue comme un «outil d'organisation» et favorise des «flottements entre vers et phrases» (phrases-paragraphes) (p. 261). C'est en définitive une «perception plus organique autant que plus personnelle de la phrase écrite» (p. 256) qui prévaut, le texte étant "désormais la seule unité stable envisageable [...]» (p. 267).

Le dernier chapitre, le plus long, «Entre pratiques standardisées et innovations ", rédigé par Gautier et Watine, est consacré aux $\mathrm{xx}^{\mathrm{e}}$ 
et $\mathrm{XIX}^{\mathrm{e}}$ siècles. La pratique épistolaire des soldats "peu-lettrés» de la Première Guerre mondiale témoigne d'une assimilation variable de l'enseignement scolaire. La première terminologie officielle (1910) laisse de côté la phrase, objet graphique et non grammatical, au profit de la proposition. Si la phrase est officialisée en 1938, la concurrence entre les deux notions subsiste encore aujourd'hui. Elle devient alors "la sédimentation symbolique du style d'un auteur, du génie d'une époque, d'une langue ou d'une nation» (p. 289). L'essor du roman oralisé dans les années 1920-1930 modifie les jugements portés sur certaines constructions, le style oral restant d'actualité dans la prose d'aujourd'hui. Prenant la place de la période, la notion de phrase «devient l'outil majeur d'analyse et d'écriture des textes» (p. 297). Au début du siècle, les «phrases extensives» de Proust ont ouvert la voie à un mouvement «d'allongement qui trouvera de nouvelles formes dans la seconde moitié du siècle» (p. 303). Alors que l'entre-deux-guerres promeut la phrase néoclassique, l'après-guerre est un «tournant majeur dans la contestation d'une syntaxe normée» (p. 313) ("point interruptif», "déponctuation", "phrase fleuve»), nouvel "étalon de la valeur d'un style individuel» (p. 320). La phrase s'émancipe de la grammaire scolaire comme du texte, devenant le «format adéquat de la modernité médiatique»(p. 327) (slogans et petites phrases), parallèlement au retour de la rhétorique. Depuis quelques décennies, la révolution numérique affecte «l'existence même de la phrase en tant qu'unité de segmentation du discours écrit» (p. 344), caractérisée par une «association de plus en plus étroite de signes linguistiques et d'images» (p. 348).

Face à la riche bibliographie en fin de volume, on peut regretter le parti-pris de citations non référencées, bien qu'il apparaisse cohérent avec la nature du projet et la cible de l'ouvrage. Si le propos s'écarte souvent de la phrase à proprement parler, c'est pour mieux cerner ses spécificités, son statut et sa valeur. À cet égard, étant donné que l'ouvrage englobe l'objet empirique et la notion théorisée, peut-être la théorisation de la phrase aurait-elle pu être également évoquée pour le $\mathrm{xx}^{\mathrm{e}}$ siècle, qui voit se développer tant les descriptions grammaticales $\mathrm{du}$ français que les théories linguistiques. La palme de l'effort pédagogique, sensible entre autres dans la manière d'établir des ponts entre les chapitres, reviendrait plutôt aux chapitres d'ouverture et de clôture. En somme, ce volume riche et soigné offre une mise en perspective 
synthétique précieuse tant pour les amateurs de littérature et de langue françaises que pour les étudiants et les spécialistes.

Sophie JOLLIN-BERTOCCHI Université Paris-Saclay - UVSQ, CHCSC sophie.bertocchi-jollin@uvsq.fr

\section{Références}

BRUNOT F. (1905-1953), Histoire de la langue française des origines à 1900, Paris, A. Colin.

MARCHELLO-NIZIA C., COMBETTES B., PRÉVOST S. \& SCHEER T. (éds) (2020), Grande Grammaire Historique du Français, vol. 1 et 2, Berlin, De Gruyter Mouton.

REY A., DUVAL F. \& SIOUFFI G. (éds.) (2007), Mille ans de langue française: histoire d'une passion, Paris, Perrin. 